\title{
Correlation Analysis Algorithm-Based Multiple-Input Single-Output Wiener Model with Output Noise
}

\author{
Li Jia $\mathbb{D}^{1},{ }^{1}$ Yangyang $\mathrm{Li}\left(\mathbb{D},{ }^{1}\right.$ and Feng $\mathrm{Li}^{2}$ \\ ${ }^{1}$ Shanghai Key Laboratory of Power Station Automation Technology, Department of Automation, \\ College of Mechatronics Engineering and Automation, Shanghai University, Shanghai 200072, China \\ ${ }^{2}$ College of Electrical and Information Engineering, Jiangsu University of Technology, Changzhou 213001, China
}

Correspondence should be addressed to Li Jia; jiali@staff.shu.edu.cn

Received 18 April 2019; Revised 30 July 2019; Accepted 11 October 2019; Published 12 November 2019

Academic Editor: Giacomo Innocenti

Copyright (c) $2019 \mathrm{Li}$ Jia et al. This is an open access article distributed under the Creative Commons Attribution License, which permits unrestricted use, distribution, and reproduction in any medium, provided the original work is properly cited.

\begin{abstract}
A novel identification algorithm for the correlation analysis-based multiple-input single-output (MISO) neurofuzzy Wiener model with noise is proposed. Firstly, several sets of Gaussian signals are utilized to realize the decoupling between the dynamic linear blocks and the static nonlinear blocks of a MISO Wiener system. Then, the correlation analysis is adopted to identify the parameters of the linear parts, and the problem that the output of static nonlinear block is immeasurable can be solved. As a result, it can circumvent the problem of initialization and convergence of the model parameters encountered by the existing iterative approach used for the identification of the Wiener model. Next, the least-squares algorithm is employed to estimate the consequent parameters of the nonlinear blocks which can avoid the influence of noise. Finally, examples are used to illustrate the performance of the proposed method.
\end{abstract}

\section{Introduction}

Wiener model consists of the cascade of a dynamic linear block followed by a static nonlinear block which can approximate a lot of industry processes, such as $\mathrm{PH}$ neutralization [1], continuous stirred tank reactor (CSTR) [2], heat exchanger [3], and distillation columns [4]. Thus, blockoriented models have gained wide attention in the system identification and automatic control fields [5-8].

Up to now, plenty of methodologies have been used to identify block-oriented models. In general, the identification approaches based on input-output signals for block-oriented models can be divided into two parts: the synchronous method and the separate step algorithm. The synchronous method identifies the block-oriented system by directly constructing the hybrid model of the dynamic linear block and the static nonlinear block [9], such as the direct identification method [10], the subspace algorithm [11], the overparameterization approach [12], and the hierarchical identification methods [13]. The separate step algorithm decomposes the block-oriented model into the dynamic linear block and the static nonlinear block, such as the correlation method [14], the iterative algorithm [15], the blind approach [16], and stochastic technique [17].

Few papers concentrated on the MISO Wiener model. Guo and Papanicolaou transformed the MISO Wiener model into a pseudolinear system, and then, the adaptive recursive pseudolinear regressions and the smoothing technique were employed to estimate the intermediate variables so that the parameters of the MISO Wiener model were estimated [18]. Tiels et al. [19] formulated the dynamical linear part of the MISO Wiener-Schetzen model with a set of orthonormal basis functions, and the system poles were estimated by the best linear approximation algorithm. Feroldi, Gomez, and Roda introduced a MISO Wiener model identification method based on support vector (SV) regression which is used to estimate a nonlinear dynamical model for the air supply system of laboratory polymer electrolyte membrane fuel cells [20]. However, the abovementioned methods assume that the static nonlinear block is of the polynomial model, which has a lot of limitations. If the nonlinearity is discontinuous or not in the 
polynomial form, the algorithms do not converge. Moreover, high-degree polynomials have oscillatory behaviour. Zhou et al. developed a gradient-based iterative identification approach for the MISO Wiener model which can approximate the glutamate fermentation process [21]. In 2011, a prediction error identification principle for the MISO Wiener model was proposed by Silva to solve the problem in anesthesia [22]. A hybrid genetic and swarming intelligencebased hierarchical cultural algorithm was applied to identify the MISO Wiener model in [23]. But the aforementioned results considered the identification of the MISO Wiener model without output noise, which is not the case in the real industrial processes.

A useful theorem which is summarized in Bussgang's classic principle (see [24]) about Gaussian signal presents that $R_{v u}(\tau)=b_{0} R_{u}(\tau)$ holds for an arbitrary static nonlinearity if the input signal is separable, where $R_{v u}(\tau)=$ $E(v(k) u(k-\tau))$ is the cross-correlation between the input signal $u(t)$ and the output signal $v(t), R_{u}(\tau)=E(u(k) u(k-$ $\tau))$ is the autocorrelation of the input signal, and $b_{0}=E\left(f^{\prime}(u(k))\right)$, where $f^{\prime}(\cdot)$ is the first-order derivative of $f(\cdot)$. Nuttall generalized this theorem to a larger kind of separable signals, such as sine signals, random binary signals, and several kinds of modulated signals [25]. Enqvist and Ljung further proposed a principle: the process is separable if and only if the condition expectation of the signal $u(k)$ satisfies $E(u(k-\tau) \mid u(k))=a(\tau) u(k)$, where $a(\tau)=R_{u}(\tau) / R_{u}(0)$ [26]. Greblicki and Pawlak studied the white noise incentive signal-based identification algorithm for the Hammerstein model in [27]. Goethals et al. generalized the linear subspace method and combined correlation analysis with least squares support vector machines (LSSVM) regression to estimate the parameters of the dynamics and nonlinearity representation of the HammersteinWiener model [28]. In our previous works, Jia et al. extended Bussgang's theorem to Hammerstein model [29-31], and the correlation analysis algorithm was applied to the SISO Wiener model in [32]. However, most of the real industry processes [33-35] are inherently multivariable systems which can describe the relationships among different variables more accurately. Thus, multivariable nonlinear identification techniques are required. In addition, according to [36-38], sometimes for reason of making the system as close as possible to the real one, MISO block-oriented models with distributed dynamic linear blocks are chosen to represent some nonlinear industry processes. Therefore, motivated by our previous works, a novel identification algorithm for correlation analysis algorithm-based MISO-distributed Wiener model with noise is presented. The nonlinearities of the MISO Wiener model are approximated by neurofuzzy models, which can avoid the inevitable restrictions on static nonlinearities encountered by using the polynomial models. Gaussian signals are adopted to separate the dynamic linear blocks and the static nonlinear blocks. Then, the correlation analysis is employed for the identification of the dynamic linear parts so as to obtain the intermediate variables indirectly. At last, least squares approach is used to identify the nonlinearity and the estimation is proved to be unbiased.
The rest of the paper is organized as follows. The identification problem of the MISO neurofuzzy Wiener model with noise is described in Section 2. A correlation analysis-based identification approach is presented in detail in Section 3. Section 4 gives the simulation examples, followed by the conclusion given in Section 5 .

\section{MISO Wiener Neurofuzzy Model with Noise}

Consider a MISO Wiener model (see [18]) with noise which consists of the dynamic linear blocks and the static nonlinear blocks as shown in Figure 1 as given by

$$
\begin{aligned}
v_{i}(t) & =\frac{B_{i}(q)}{A_{i}(q)} u_{i}(t), \\
y_{i}(t) & =f_{i}\left(v_{i}(t)\right), \\
y(t) & =\sum_{i=1}^{m} y_{i}(t)+\eta(t), \quad i=1,2, \ldots, m, \\
A_{i}(q) & =1+a_{i 1} q^{-1}+a_{i 2} q^{-2}+\cdots+a_{\mathrm{in}_{a}} q^{-n_{a}}, \\
B_{i}(q) & =1+b_{i 1} q^{-1}+b_{i 2} q^{-2}+\cdots+b_{\mathrm{in}_{b}} q^{-n_{b}},
\end{aligned}
$$

where $u_{i}(t)(i=1,2, \ldots, m)$ and $y(t)$ which can be measured represent the input and output processes at the $t$-th sampling time, respectively. $i$ denotes the $i$-th branch of the MISO Wiener model, $i=1,2, \ldots, m . v_{i}(t)(i=1,2, \ldots, m)$ corresponds to the intermediate variable; $f_{i}\left(v_{i}(t)\right)(i=1,2, \ldots, m)$ is the static nonlinearity of the $i$-th branch of the MISO Wiener model. $y_{i}(t)(i=1,2, \ldots, m)$ which cannot be measured is the output of the nonlinear block. $\eta(t)$ denotes the white noise, $q^{-1}$ is the backward shift, and $n_{i a}$ and $n_{i b}$ are integers concerned with the model order.

In this paper, the target of the proposed identification approach is to obtain a MISO Wiener model such that the following cost function $E$ is made acceptable small, that is,

$$
\begin{aligned}
& E\left(\widehat{A}_{1}, \widehat{A}_{2}, \ldots, \widehat{A}_{m}, \widehat{B}_{1}, \widehat{B}_{2}, \ldots, \widehat{B}_{m}, \widehat{f}_{1}\left(\widehat{v}_{1}(t)\right),\right. \\
& \left.\widehat{f}_{2}\left(\widehat{v}_{2}(t)\right), \ldots, \widehat{f}_{m}\left(\widehat{v}_{m}(t)\right)\right) \\
& \quad=\frac{1}{2 N_{P}} \sum_{t=1}^{N_{P}}(\hat{y}(t)-y(t))^{2} \leq \varepsilon,
\end{aligned}
$$

s.t.

$$
\begin{aligned}
\widehat{v}_{i}(t) & =\frac{\widehat{B}_{i}(q)}{\widehat{A}_{i}(q)} u_{i}(t), \\
\widehat{y}_{i}(t) & =\widehat{f}_{i}\left(\widehat{v}_{i}(t)\right), \quad i=1,2, \ldots, m, \\
\hat{y}(t) & =\sum_{i=1}^{m} \widehat{y}_{i}(t)+e(t),
\end{aligned}
$$

where $\varepsilon$ is a given tolerance, $\widehat{f}(\cdot)$ is the estimated nonlinear function, $\hat{y}(t)$ is the estimated output of the MISO Wiener model, $\widehat{v}_{i}(t)$ is the estimated intermediate variable of $i$-th 


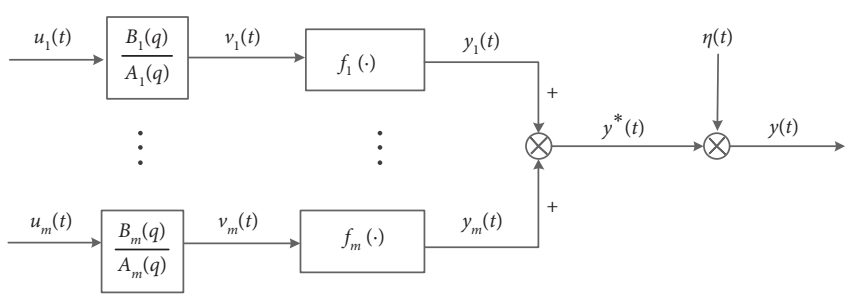

Figure 1: The structure of the MISO Wiener model.

branch, $N_{P}$ is the number of the input and output data, and " $\wedge$ " represents "model".

As shown in Figure 2, the nonlinear function of each branch of the MISO Wiener model is represented by a fourlayer neurofuzzy system which integrates the Takagi-Sugeno fuzzy system and radial basis function-based feed-forward network into a connection structure. The first layer is the input layer in which the nodes just transmit the input signal to the next layer. The second layer is the membership layer where the membership function of the input variable is calculated. The third layer is the fuzzy rule layer, and the number of the nodes in this layer denotes the fuzzy rules. The last layer is the output layer. The output of the $i$-th neurofuzzy model is given by

$$
\hat{y}_{i}(t)=\sum_{j=1}^{N_{i}} \phi_{i j}\left(\widehat{v}_{i}(t)\right) w_{i j}, \quad i=1,2, \ldots, m,
$$

where

$$
\phi_{i l}\left(\widehat{v}_{i}(t)\right)=\frac{\exp \left(-\left(\left(\widehat{v}_{i}(t)-c_{i l}\right)^{2} / \sigma_{i l}^{2}\right)\right)}{\sum_{l=1}^{N_{i}} \exp \left(-\left(\left(\widehat{v}_{i}(t)-c_{i l}\right)^{2} / \sigma_{i l}^{2}\right)\right)},
$$

where $c_{i l}$ and $\sigma_{i l}$ denote the center and width of the membership function, respectively, $w_{i l}$ is the consequent parameters of the neurofuzzy model, and $N_{i}$ represents the fuzzy rule of the nonlinear block of the $i$-th branch.

\section{Identification of Correlation Analysis-Based MISO Wiener Model}

Gaussian signals with independent and identical distribution are adopted to separate the linear blocks from the nonlinear blocks by this proposed algorithm so that the immeasurable

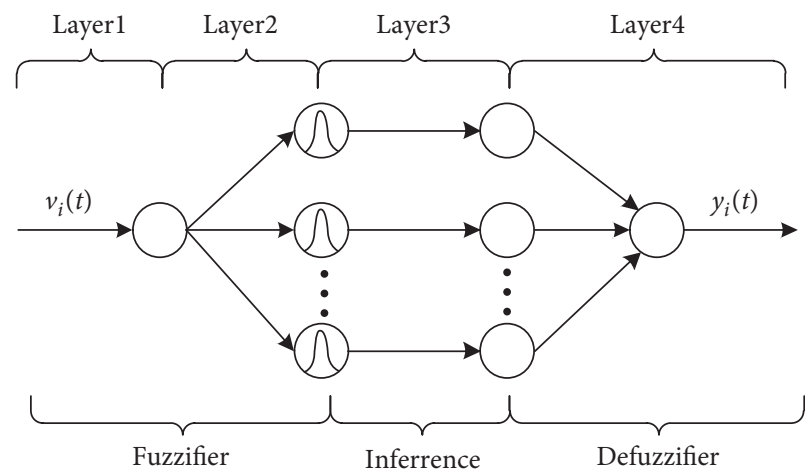

Figure 2: The structure of the neurofuzzy model.

intermediate variable $v_{i}(t)(i=1,2, \ldots, m)$ is obtained indirectly.

Assume that the input and output of the model are zero initial state and the input signals are Gaussian sequences with independent and identical distribution, i.e., $u_{i}(t) \sim$ $\left(0, \sigma_{u_{i}}^{2}\right)(i=1,2, \ldots, m)$. And presume that the cross-correlations between different input signals are zero. Then, the intermediate variable $v_{i}(t)$ of the MISO Wiener model can be written as follows:

$$
\begin{aligned}
v_{i}(1) & =0, \\
v_{i}(2) & =b_{i 1} u_{i}(1), \\
v_{i}(3) & =-a_{i 1} v_{i}(2)-a_{i 2} v_{i}(1)+b_{i 1} u_{i}(2)+b_{i 2} u_{i}(1), \\
& =\left(-a_{i 1} b_{i 1}+b_{i 2}\right) u_{i}(1)+b_{i 1} u_{i}(2), \ldots, m, \\
\ldots & \\
v_{i}(t) & =\sum_{k=1}^{t-1} h_{i, t}(k) u_{i}(t-k),
\end{aligned}
$$

where $h_{i, t}(k)$ denotes the coefficient of $u_{i}(t-k)$ of the $i$-th branch at $t$-th time.

Then, the conditional expectation of $v_{i}(t)$ can be deduced:

$$
\begin{aligned}
E\left(v_{i}(t-\tau) \mid v_{i}(t)\right) & =\sum_{k=1}^{t-\tau-1} h_{i, t-\tau}(k) E\left(u_{i}(t-\tau-k) \mid \sum_{\xi=1}^{t-1} h_{i, t}(\xi) u_{i}(t-\xi)\right) \\
& =\sum_{k=1}^{t-\tau-1} h_{i, t-\tau}(k) E\left(u_{i}(t-\tau-k) \mid h_{i, t}(\tau+k) u_{i}(t-\tau-k)+\sum_{\xi=1}^{t-1} h_{i, t}(\xi) u_{i}(t-\xi), \xi \neq \tau+k\right) \\
& =\sum_{k=1}^{t-\tau-1} h_{i, t-\tau}(k) E\left(U_{i, t-\tau-k} \mid X_{i, t-\tau-k}+Y_{i, t}\right),
\end{aligned}
$$


where $\quad U_{i, t-\tau-k}=u_{i}(t-\tau-k), \quad X_{i, t-\tau-k}=h_{i, t}(\tau+k) u_{i}(t-$ $\tau-k)$, and $Y_{i, t}=\sum_{\xi=1}^{t-1} h_{i, t}(\xi) u_{i}(t-\xi), \xi \neq \tau+k$. Assume that the input signals are Gaussian signals with independent and identical distribution, a linear combination of a finite number of Gaussian signal is necessarily also Gaussian signal; thus, $v_{i}(t)$ subjects to normal distribution with zero means and variance $\sigma_{v_{i}}^{2}=\sigma_{u_{i}}^{2} \sum_{k=1}^{t-1} h_{i, t}^{2}(k)$, i.e., $v_{i}(t) \sim\left(0, \sigma_{v_{i}}^{2}\right)$.
$Y_{i, t}$ subjects to normal distribution with zero means and variance $\sigma_{Y_{i}}=\sigma_{u_{i}}^{2} \sum_{\xi=1}^{t-1} h_{i, t}^{2}(\xi), \xi \neq \tau+k$, i.e., $Y_{i, t} \sim\left(0, \sigma_{Y_{i}}^{2}\right)$.

As can be seen from (7), $U_{i, t-\tau-k}$ and $X_{i, t-\tau-k}$ are independent of $Y_{i, t}$, respectively. Moreover, we can get $U_{i, t-\tau-k}=X_{i, t-\tau-k} / h_{i, t}(\tau+k)$. And assume that $V_{i, t}=v_{i}(t)=X_{i, t-\tau-k}+Y_{i, t}$, and thus, the joint probability density function of $U_{i, t-\tau-k}$ and $V_{i, t}$ is as follows:

$$
\begin{aligned}
g_{\left(U_{i, t-\tau-k}, V_{i, t}\right)}\left(u_{i}, v_{i}\right) & =g_{\left(X_{i, t-\tau-k}, Y_{i, t}\right)}\left(h_{i, t}(\tau+k) u_{i}, v_{i}-h_{i, t}(\tau+k) u_{i}\right) \times|J a c|^{-1} \\
& =g_{X_{i, t-\tau-k}}\left(h_{i, t}(\tau+k) u_{i}\right) \times g_{Y_{i, t}}\left(v_{i}-h_{i, t}(\tau+k) u_{i}\right) \times|J \mathrm{ac}|^{-1} \\
& =\frac{1}{\sqrt{2 \pi} h_{i, t}(\tau+k) \sigma_{u_{i}}} \times e^{-\left(\left(h_{i, t}(\tau+k) u_{i}\right)^{2} / 2\left(h_{i, t}(\tau+k) \sigma_{u_{i}}\right)^{2}\right)} \times \frac{1}{\sqrt{2 \pi} \sigma_{Y_{i}}} \times e^{-\left(\left(v_{i}-h_{i, t}(\tau+k) u_{i}\right)^{2} / 2 \sigma_{Y_{i}}^{2}\right)} \times h_{i, t}(\tau+k) \\
& =\frac{1}{2 \pi \sigma_{u_{i}} \sigma_{Y_{i}}} e^{-\left(u_{i}^{2} \sigma_{Y_{i}}^{2}+\left(v_{i}-h_{i, t}(\tau+k) u_{i}\right)^{2} \sigma_{u_{i}}^{2} / 2 \sigma_{u_{i}}^{2} \sigma_{Y_{i}}^{2}\right)}
\end{aligned}
$$

where Jac is the Jacobian determinant, that is (see [39]),

$$
\mathrm{Jac}=\frac{\partial\left(U_{i, t-\tau-k}, V_{i, t}\right)}{\partial\left(X_{i, t-\tau-k}, Y_{i, t}\right)}=\left|\begin{array}{ll}
\frac{\partial U_{i, t-\tau-k}}{\partial X_{i, t-\tau-k}} & \frac{\partial U_{i, t-\tau-k}}{\partial Y_{i, t}} \\
\frac{\partial V_{i, t}}{\partial X_{i, t-\tau-k}} & \frac{\partial V_{i, t}}{\partial Y_{i, t}}
\end{array}\right|=\frac{1}{h_{i, t}(\tau+k)} .
$$

Thus, the conditional probability density function of $U_{i, t-\tau-k}$ is given by

$$
\begin{aligned}
g_{U_{i, t-\tau-k} \mid V_{i, t}=C}\left(u_{i} \mid v_{i}=c\right) & =\frac{g_{\left(U_{i, t---k}, V_{i, t}\right.}\left(u_{i}, c\right)}{g_{V_{i, t}}(c)} \\
& =\frac{\sigma_{v_{i}}}{\sqrt{2 \pi} \sigma_{u_{i}} \sigma_{Y_{i}}} \times e^{\left(c^{2} / 2 \sigma_{v_{i}}^{2}\right)-\left(\left(u_{i}^{2} \sigma_{Y_{i}}^{2}+\left(v_{i}-h_{i, t}(\tau+k) u_{i}\right)^{2} \sigma_{u_{i}}^{2}\right) / 2 \sigma_{u_{i}}^{2} \sigma_{Y_{i}}^{2}\right)} \\
& =k_{c} e^{-\left(\left(k_{a} u_{i}^{2}-2 k_{b} c u_{i}\right) / 2 \sigma_{u_{i}}^{2} \sigma_{Y_{i}}^{2}\right)} \\
& =k_{d} e^{-\left(k_{a}\left(u_{i}-k_{b} c / k_{a}\right)^{2} / 2 \sigma_{u_{i}}^{2} \sigma_{Y_{i}}^{2}\right)},
\end{aligned}
$$

where the coefficients are defined in the following equation and the constant $c$ is the value of $v_{i}(t)$ :

$$
\begin{aligned}
& k_{a}=\left(\sigma_{Y_{i}}^{2}+\sigma_{u_{i}}^{2} h_{i, t}(\tau+k)^{2}\right), \\
& k_{b}=\sigma_{u_{1}}^{2} h_{i, t}(\tau+k), \\
& k_{c}=\frac{\sigma_{v_{i}}}{\sqrt{2 \pi} \sigma_{u_{i}} \sigma_{Y_{i}}} e^{\left(\sigma_{Y_{i}}^{2}-\sigma_{v_{i}}^{2}\right) c^{2} / 2 \sigma_{v_{i}}^{2} \sigma_{Y_{i}}^{2}}, \\
& k_{d}=k_{c} \cdot e^{k_{b}^{2} c^{2} / 2 k_{a} \sigma_{u_{i}}^{2} \sigma_{Y_{i}}^{2}} .
\end{aligned}
$$

As a result, the conditional expectation of $u_{i}(t-\tau-k)$ is given by

$$
E\left(u_{i}(t-\tau-k) \mid \sum_{\xi=1}^{t-1} h_{i, t}(\xi) u_{i}(t-\xi)=c\right)=\frac{k_{b}}{k_{a}} c,
$$

From (7) and (12), we can obtain the following equation:

$$
E\left(v_{i}(t-\tau) \mid v_{i}(t)\right)=\frac{k_{b}}{k_{a}} \cdot c \cdot \sum_{k=1}^{t-\tau-1} h_{i, t-\tau}(k)=a_{i}(\tau) \cdot c,
$$


where $a_{i}(\tau)=\left(k_{b} / k_{a}\right) \cdot \sum_{k=1}^{t-\tau-1} h_{i, t-\tau}(k), i=1,2, \ldots, m$ is a constant. In conclusion, according to [26], the intermediate variable $v_{i}(t)$ satisfies the separability of the process.

Theorem 1. For a MISO Wiener model as expressed in (1), if the input signals are Gaussian signals with independent identical distribution and the expectations of inputs satisfy $E\left(u_{i}(t)\right)=0(i=1,2, \ldots, m)$, the following equation holds:

$$
\mathbf{R}_{y u}=\mathbf{B R}_{u},
$$

where $\quad \mathbf{R}_{y u}=\left[R_{y u_{1}}, R_{y u_{2}}, \ldots, R_{y u_{m}}\right]^{T}, \quad \mathbf{B}=\operatorname{diag}\left(b_{01} L_{1}\right.$, $\left.b_{02} L_{2}, \ldots, b_{0 m} L_{m}\right)$, and $\mathbf{R}_{u}=\left[R_{u_{1}}, R_{u_{u}}, \ldots, R_{u_{m}}\right]^{T}$, and $R_{y u_{i}}(\tau)$ denotes the cross-correlation function between $y(t)$ and the $i$-th input $u_{i}(t), R_{u_{i}}(\tau)$ represents the autocorrelation of $u_{i}(t), L_{i}(q)$ is the linear part, i.e., $L_{i}(q)=B_{i}(q) / A_{i}(q)$, and $b_{0 i}=E\left(y_{i}(t) v_{i}(t)\right) / E\left(v_{i}(t) v_{i}(t)\right)$ is a constant.

Proof. As can be seen from (13), when the input is Gaussian signal, the output $v_{i}(t)$ of the dynamic of the $i$-th branch satisfies the separability of the process. Thus, for a MISO Wiener model, we can get the following equation:

$$
\begin{aligned}
R_{y v_{i}}(\tau)= & E\left(y(t) v_{i}(t-\tau)\right) \\
= & E\left(\left(y_{1}(t)+y_{2}(t)+\cdots+y_{m}(t)+\eta(t)\right) v_{i}(t-\tau)\right) \\
= & E\left(y_{1}(t) v_{i}(t-\tau)\right)+E\left(y_{2}(t) v_{i}(t-\tau)\right) \\
& +\cdots+E\left(y_{m}(t) v_{i}(t-\tau)\right) \\
& +E\left(\eta(t) v_{i}(t-\tau)\right), \quad i=1,2, \ldots, m \\
= & E\left(y_{i}(t) v_{i}(t-\tau)\right) \\
= & E\left(y_{i}(t) E\left(v_{i}(t-\tau) \mid v_{i}(t)\right)\right) \\
= & b(\tau) E\left(y_{i}(t) v_{i}(t)\right) .
\end{aligned}
$$

From (15), we know that the cross-correlation function between $v_{i}(t)$ and the immeasurable variable $y_{i}(t)$ can be replaced by the cross-correlation function between $v_{i}(t)$ and $y(t)$ :

$$
\begin{aligned}
R_{v_{i}}(\tau) & =E\left(v_{i}(t) v_{i}(t-\tau)\right) \\
& =E\left(v_{i}(t) E\left(v_{i}(t-\tau) \mid v_{i}(t)\right)\right) \\
& =b(\tau) E\left(v_{i}(t) v_{i}(t)\right) .
\end{aligned}
$$

According to (15) and (16), the following equation holds:

$$
R_{y v_{i}}(\tau)=\frac{E\left(y_{i}(t) v_{i}(t)\right)}{E\left(v_{i}(t) v_{i}(t)\right)} R_{v_{i}}(\tau)=b_{0 i} R_{v_{i}}(\tau) .
$$

According to [40], for a stable and time-invariant linear system, its impulse response can be represented by

$$
L_{i}(q)=\sum_{k=1}^{\infty} l_{i}(k) q^{-k}
$$

Thus, the output of the dynamic linear block of $i$-th branch can be written as
$v_{i}(t)=L_{i}(q) u_{i}(t)=\sum_{k=1}^{\infty} l_{i}(k) q^{-k} \cdot u_{i}(t)=\sum_{k=1}^{\infty} l_{i}(k) u_{i}(t-k)$.

And (15) and (16) can be rewritten as follows:

$$
\begin{aligned}
R_{y v_{i}}(\tau) & =E\left(y(t) v_{i}(t-\tau)\right) \\
& =E\left(y(t) \sum_{k=1}^{\infty} l_{i}(k) u_{i}(t-\tau-k)\right) \\
& =\sum_{k=1}^{\infty} l_{i}(k) R_{y u_{i}}(\tau+k) \\
& =\sum_{k=1}^{\infty} l_{i}(k) q^{k} \cdot R_{y u_{i}}(\tau), \\
R_{v_{i}}(\tau) & =E\left(v_{i}(t) v_{i}(t-\tau)\right) \\
& =E\left(\sum_{k=1}^{\infty} l_{i}(k) u_{i}(t-k) \sum_{\zeta=1}^{\infty} l_{i}(\varsigma) u_{i}(t-\tau-\varsigma)\right) \\
& =\sum_{k=1}^{\infty} \sum_{\zeta=1}^{\infty} l_{i}(k) R_{u_{i}}(\tau+\varsigma-k) l_{i}^{T}(\varsigma) \\
& =\sum_{\varsigma=1}^{\infty} l_{i}(\varsigma) q^{\xi} \cdot R_{u_{i}}(\tau) \cdot \sum_{k=1}^{\infty} l_{i}(k) q^{-k} .
\end{aligned}
$$

Then, by substituting (20) and (21) into (17), we can get the following equation:

$$
\sum_{k=1}^{\infty} l_{i}(k) q^{k} \cdot R_{y u_{i}}(\tau)=b_{0 i} \sum_{\zeta=1}^{\infty} l_{i}(\varsigma) q^{\xi} \cdot R_{u_{i}}(\tau) \cdot \sum_{k=1}^{\infty} l_{i}(k) q^{-k},
$$

where $\sum_{k=1}^{\infty} g_{i}(k) q^{k}$ on the left side of (22) is equivalent to $\sum_{\varsigma=1}^{\infty} g_{i}(\varsigma) q^{\xi}$ on the right side of (22).

Thus, we have

$$
\begin{aligned}
R_{y u_{i}}(\tau) & =b_{0 i} R_{u_{i}}(\tau) \cdot \sum_{k=1}^{\infty} l_{i}(k) q^{-k} \\
& =b_{0 i} L_{i}(q) \cdot R_{u_{i}}(\tau) .
\end{aligned}
$$

Then, (14) holds. This completes the proof.

Remark 1. In this paper, the MISO-distributed block-oriented nonlinear Wiener model which is consistent with the model in [18] is mainly considered. The content before Theorem 1 has proved that $v_{i}(t)$ satisfies the separability of the process when the input signals $u_{i}(t)$ are Gaussian signals. Thus, $R_{y_{i} v_{i}}(\tau)=b_{0 i} R_{v_{i}}(\tau)$ holds according to Bussgang's classic theorem. However, as shown in Figure 1, $v_{1}, v_{2}, \ldots, v_{m}$ and $y_{1}, y_{2}, \ldots, y_{m}$ are immeasurable variables, and $R_{y_{i} v_{i}}(\tau)=b_{0 i} R_{v_{i}}(\tau)$ cannot be used to identify the parameters of the proposed Wiener model directly. Therefore, Theorem 1 is proposed to solve this problem. The input and output relationship of the MISO Wiener model, that is $R_{y u_{i}}(\tau)=b_{0 i} L_{i}(q) \cdot R_{u_{i}}(\tau)$, is deduced so that the measurable 
variables $u_{1}, u_{2}, \ldots, u_{m}$ and $y$ can be utilized to estimate the parameters of dynamic linear blocks.

3.1. Identification of the Dynamic Linear Blocks. Correlation analysis is adopted to identify the linear blocks of the MISO Wiener model with noise.

According to Theorem 1 and (23), we have

$$
\begin{aligned}
R_{y u_{i}}(\tau)= & -\sum_{n=1}^{n_{a_{i}}} a_{\text {in }} R_{y u_{i}}(\tau-n) \\
& +b_{0 i} \sum_{j=1}^{n_{b_{i}}} b_{i j} R_{u_{i}}(\tau-j), \quad i=1,2, \ldots, m, \\
= & -\sum_{n=1}^{n_{a_{i}}} a_{\text {in }} R_{y u_{i}}(\tau-n)+\sum_{j=1}^{n_{b_{i}}} \widetilde{b}_{i j} R_{u_{i}}(\tau-j) \\
= & \boldsymbol{\Phi}_{i} \boldsymbol{\theta}_{i},
\end{aligned}
$$

where $\widetilde{b}_{i j}=b_{0 i} b_{i j}$.

Then, the following equation holds:

$$
\mathbf{R}_{y u}=\boldsymbol{\phi} \boldsymbol{\theta} .
$$

Thus, the parameters of dynamic linear block can be estimated by least squares algorithm:

$$
\boldsymbol{\theta}=\left(\boldsymbol{\Phi}^{T} \boldsymbol{\Phi}\right)^{-1} \boldsymbol{\Phi} \mathbf{R}_{y u},
$$

where $\quad \mathbf{R}_{y u}=\left[R_{y u_{1}}, R_{y u_{2}}, \ldots, R_{y u_{m}}\right]^{T}, \quad R_{y u_{i}}=\left[R_{y u_{i}}(1), R_{y u_{i}}\right.$ (2), $\left.\ldots, R_{y u_{i}}(P)\right]^{T} \in R^{P \times 1}, \quad \boldsymbol{\theta}=\left[\theta_{1}, \theta_{2}, \ldots, \theta_{m}\right]^{T}, \hat{\theta}_{i}=\left(\widehat{a}_{i 1}\right.$, $\left.\widehat{a}_{i 2}, \ldots, \widehat{a}_{\mathrm{in}_{a_{i}}} \widetilde{\widetilde{b}}_{i 1}, \widehat{\widetilde{b}}_{i 1}, \ldots, \widehat{\widetilde{b}}_{\mathrm{in}_{b_{i}}}\right)^{T} \in R^{\left(n_{a_{i}}+n_{b_{i}}\right) \times 1}, \quad \tau=1,2, \ldots$, $P\left(P \geq n_{a_{i}}+n_{b_{i}}\right), \Phi=\operatorname{diag}\left(\Phi_{1}, \Phi_{2}, \ldots, \Phi_{m}\right)$, and

$$
\begin{aligned}
\Phi_{i} & =\left[\begin{array}{ccccc}
-R_{y u_{i}}(0) & -R_{y u_{i}}(1) & -R_{y u_{i}}(2) & \cdots & -R_{y u_{i}}(P-1) \\
0 & -R_{y u_{i}}(0) & -R_{y u_{i}}(1) & \cdots & -R_{y u_{i}}(P-2) \\
\vdots & \vdots & \vdots & \ddots & \vdots \\
0 & 0 & 0 & \cdots & -R_{y u_{i}}\left(P-n_{a}\right) \\
R_{u_{i}}(0) & R_{u_{i}}(1) & R_{u_{i}}(2) & \cdots & R_{u_{i}}(P-1) \\
0 & R_{u_{i}}(0) & R_{u_{i}}(1) & \cdots & R_{u_{i}}(P-2) \\
\vdots & \vdots & \vdots & \ddots & \vdots \\
0 & 0 & 0 & \cdots & R_{u_{i}}\left(P-n_{b}\right)
\end{array}\right]^{T} \\
& \in R^{P \times\left(n_{a_{i}}+n_{b_{i}}\right)}
\end{aligned}
$$

where $R_{y u_{i}}(\tau)$ and $R_{u_{i}}(\tau)$ are as follows:

$$
\begin{aligned}
& \widehat{R}_{y u_{i}}(\tau)=\frac{1}{N} \sum_{t=1}^{N} y(t) u_{i}(t-\tau), \\
& \widehat{R}_{u_{i}}(\tau)=\frac{1}{N} \sum_{t=1}^{N} u_{i}(t) u_{i}(t-\tau) .
\end{aligned}
$$

3.2. Identification of the Static Nonlinear Blocks. After the linear blocks have been identified, the intermediate variable $\widehat{v}_{i}(t)$ can be further estimated according to the input signal $u_{i}(t)$ and the estimated parameter $\widehat{\theta}_{i}$. Next, the clustering approach used in $[41,42]$ is employed to identify the antecedent parameters of static nonlinear blocks. And the proposed algorithm focuses on the consequent parameters $w_{i j}$. From (1) and (4), we have

$$
y(t)=\mathbf{W} \psi(t)+\eta(t)
$$

where $\quad \mathbf{W}=\left[W_{1}, W_{2}, \ldots, W_{m}\right] \in R^{1 \times L}, L=\sum_{i=1}^{m} L_{i}, \quad \mathbf{W}_{i}=$ $\left[w_{i 1}, w_{i 2}, \ldots, w_{i L_{i}}\right] \in R^{1 \times L_{i}}(i=1,2, \ldots, m)$ and $\psi(t)=\left[\psi_{1}\right.$ $\left.(t), \psi_{2}(t), \ldots, \psi_{m}(t)\right]^{T} \in R^{L \times 1}, \quad \psi_{i}(t)=\left[\phi_{i 1}\left(\widehat{v}_{i}(t)\right), \phi_{i 2}\left(\widehat{v}_{i}\right.\right.$ $\left.(t)), \ldots, \phi_{i L_{i}}\left(\widehat{v}_{i}(t)\right)\right]^{T} \in R^{L_{i} \times 1} . L_{i}$ is the clustering number of nonlinear block of the $i$-th branch.

As a result, the estimation of $\mathbf{W}$ can be obtained by using least squares approach:

$$
\mathbf{W}_{\mathrm{LS}}=\left[\sum_{t=1}^{N} y(t) \boldsymbol{\psi}^{T}(t)\right]\left[\sum_{t=1}^{N} \psi(t) \boldsymbol{\psi}^{T}(t)\right]^{-1}
$$

According to (30), substitute $y(t)$ with $\mathbf{W} \psi(t)+\eta(t)$, and then (31) can be rewritten as

$$
\begin{aligned}
\mathbf{W}_{\mathrm{LS}}= & {\left[\sum_{t=1}^{N}(\mathbf{W} \psi(t)+\eta(t)) \boldsymbol{\psi}^{T}(t)\right]\left[\sum_{t=1}^{N} \psi(t) \boldsymbol{\psi}^{T}(t)\right]^{-1} } \\
= & \mathbf{W}\left[\sum_{t=1}^{N} \psi(t) \boldsymbol{\psi}^{T}(t)\right]\left[\sum_{t=1}^{N} \psi(t) \boldsymbol{\psi}^{T}(t)\right]^{-1} \\
& +\left[\sum_{t=1}^{N} \eta(t) \boldsymbol{\psi}^{T}(t)\right]\left[\sum_{t=1}^{N} \boldsymbol{\psi}(t) \boldsymbol{\psi}^{T}(t)\right]^{-1} \\
= & \mathbf{W}+\left[\sum_{t=1}^{N} \eta(t) \boldsymbol{\psi}^{T}(t)\right]\left[\sum_{t=1}^{N} \boldsymbol{\psi}(t) \boldsymbol{\psi}^{T}(t)\right]^{-1}
\end{aligned}
$$
have

$$
\begin{aligned}
E\left(\mathbf{W}_{\mathrm{LS}}\right) & =E(\mathbf{W})+E\left(\left[\sum_{t=1}^{N} e(t) \boldsymbol{\psi}^{T}(t)\right]\left[\sum_{t=1}^{N} \psi(t) \boldsymbol{\psi}^{T}(t)\right]^{-1}\right) \\
& =\mathbf{W}+\left[\sum_{t=1}^{N} E(\eta(t)) \boldsymbol{\psi}^{T}(t)\right]\left[\sum_{t=1}^{N} \psi(t) \boldsymbol{\psi}^{T}(t)\right]^{-1} \\
& =\mathbf{W} .
\end{aligned}
$$

In conclusion, $\mathbf{W}_{\mathrm{LS}}$ is the unbiased estimation of $\mathbf{W}$.

The identification algorithm for the correlation analysisbased MISO Wiener model is concluded as follows:

Step 1 . The parameters $a_{i j}$ and $b_{i j}$ of the dynamic linear blocks of the MISO Wiener model are estimated according to (26)-(29) in Section 3.1

Step 2. The antecedent parameters $c_{i l}$ and $\sigma_{i l}$ are obtained by using the clustering approach $[41,42]$ 
Step 3. The consequent parameter $\mathbf{W}$ of each static nonlinear block is identified according to (30) and (31) in Section 3.2

\section{Examples}

4.1. Example 1. Consider the following TISO (two-input single-output) Wiener model which has two branches (see [18] which is a classical MISO-distributed Wiener model):

$$
\begin{aligned}
& v_{1}(t)=\frac{0.04308 q^{-1}+0.0315 q^{-2}}{1-1.3139 q^{-1}+0.3886 q^{-2}} u_{1}(t), \\
& y_{1}(t)=2 \tanh \left(v_{1}(t)\right)-2 \exp \left(0.1 v_{1}(t)\right)+2, \\
& v_{2}(t)=\frac{0.0305 q^{-1}+0.0254 q^{-2}}{1-1.5218 q^{-1}+0.5778 q^{-2}} u_{2}(t), \\
& y_{2}(t)= \begin{cases}v_{2}(t), & v_{2}(t) \leq 1.5, \\
1.5 \exp \left(v_{2}(t)-1.5\right), & v_{2}(t)>1.5,\end{cases} \\
& y(t)=y_{1}(t)+y_{2}(t)+\eta(t),
\end{aligned}
$$

where the white noise with zero means $\eta(t)$ is dependent on $u_{1}(t)$ and $u_{2}(t)$, respectively.

Define the noise-to-signal ratios as $\delta_{\text {ns }}=$ $\sqrt{\operatorname{var}(e(t)) / \operatorname{var}(y(t)-e(t))} \times 100 \%$ and the estimation error $\delta=\|\hat{\theta}-\theta\| /\|\theta\|$ of the linear block.

In order to identify the above TISO Wiener model, the following signals as shown in Figure 3 are adopted: (1) Gaussian signal $u_{1}(t)$ with zero means and variance 4 ; (2) Gaussian signal $u_{2}(t)$ with zero means and variance 4 ; and (3) the corresponding output signal $y(t)$.

At first, $u_{1}(t), u_{2}(t)$, and $y(t)$ are employed to estimate the parameters of linear blocks of two branches according to (25) and (29) in Section 3.1. And the modifying coefficients are $\quad b_{01}=E(y(t) v(t)) / E(v(t) v(t))=0.4517 \quad$ and $b_{02}=E(y(t) v(t)) / E(v(t) v(t))=0.3643$, respectively. Tables 1 and 2 give the results of identifying the linear parts of two branches by the proposed algorithm and the method in [18] under different noise-to-signal ratios. The estimation errors of two linear blocks are illustrated in Figures 4 and 5, respectively. It is obvious that the proposed algorithm results in a better approximation of the linear blocks than the method in [18] according to Tables 1 and 2 as well as Figures 4 and 5.

Next, the intermediate variables $\widehat{v}_{1}(t)$ and $\hat{v}_{2}(t)$ are estimated by using the input signals $u_{1}(t)$ and $u_{2}(t)$ and the estimated parameters of linear blocks. Then, the antecedent parameters of two nonlinear blocks are identified by using the clustering algorithm according to the following design parameters [41, 42]: $S_{01}=0.98, \rho_{1}=1.61, \lambda_{1}=0.02$ and $S_{02}=0.988, \rho_{2}=1.05, \lambda_{2}=0.1$, which result in two neurofuzzy models whose rules are 6 and 80, respectively. Moreover, the consequent parameters $\mathbf{W}_{1}$ and $\mathbf{W}_{2}$ of two nonlinear blocks are obtained according to (30) and (31). The polynomial method described in [18] is also constructed by using the identical data to identify the nonlinear blocks.
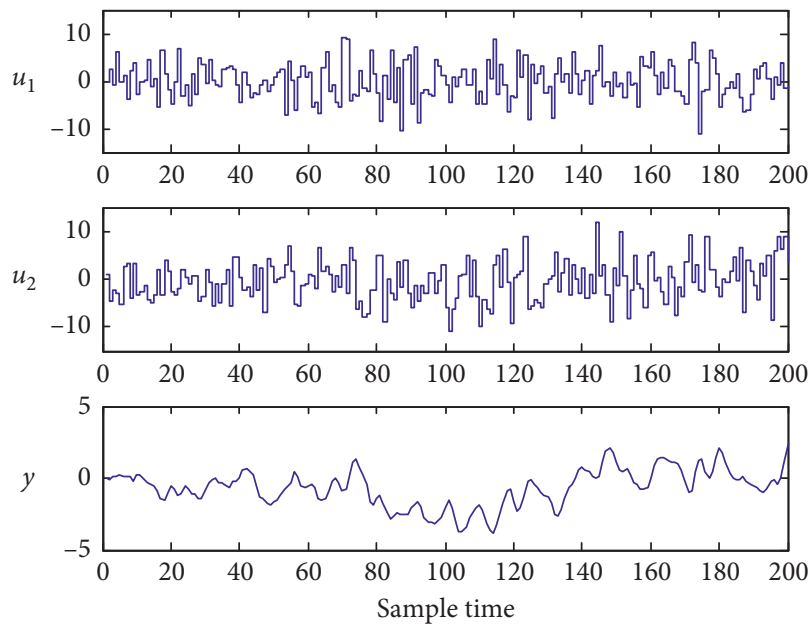

Figure 3: Part of the training data used in Example 1.

The comparisons between the proposed algorithm and the method in [18] are shown in Figures 6 and 7. The MSEs of two neurofuzzy models are $3.5 \times 10^{-3}$ and $2.0 \times 10^{-3}$, respectively, while the MSEs of two polynomial functions are $2.1 \times 10^{-2}$ and $4.1 \times 10^{-2}$, respectively. As illustrated in Figures 6 and 7, the proposed algorithm has better identification accuracy for the nonlinearity of the actual process.

4.2. Example 2. To further verify the performance of the proposed algorithm, this example considers a TISO Wiener model whose nonlinearities are two more complicated discontinuous segment nonlinear functions:

$$
\begin{aligned}
& v_{1}(t)=\frac{0.4 q^{-1}}{1+0.8 q^{-1}} u_{1}(t), \\
& y_{1}(t)= \begin{cases}2-\cos \left(3 v_{1}(t)\right)-\exp \left(-v_{1}(t)\right), & v_{1}(t) \leq 1, \\
-1, & v_{1}(t)>1,\end{cases} \\
& v_{2}(t)=\frac{0.6 q^{-1}}{1+0.3 q^{-1}} u_{2}(t), \\
& y_{2}(t)=x(t)= \begin{cases}\tanh \left(2 v_{2}(t)\right), & v_{2}(t) \leq 1.5, \\
-\frac{\exp \left(v_{2}(t)-1\right)}{\exp \left(v_{2}(t)+1\right)}, & v_{2}(t)>1.5,\end{cases} \\
& y(t)=y_{1}(t)+y_{2}(t)+\eta(t) .
\end{aligned}
$$

In order to identify the above TISO Wiener model, the following signals as shown in Figure 8 are adopted: (1) Gaussian signal $u_{1}(t)$ with zero means and variance 1.5 ; (2) Gaussian signal $u_{2}(t)$ with zero means and variance 1.9; and (3) the corresponding output signal $y(t)$.

Firstly, correlation analysis theorem in Section 3.1 is employed to identify the linear blocks, so the estimated linear blocks are $\widehat{v}_{1}(t)=-0.8052 \widehat{v}_{1}(t-1)+0.3840 u_{1}(t-1)$ 
TABLE 1: Identification results of the linear block in the first branch under different $\delta_{\text {ns }}$.

\begin{tabular}{|c|c|c|c|c|c|c|c|c|c|c|}
\hline \multirow[b]{2}{*}{$t$} & \multicolumn{5}{|c|}{ The proposed method } & \multicolumn{5}{|c|}{ The method in [18] } \\
\hline & $\widehat{a}_{11}$ & $\widehat{a}_{12}$ & $\widehat{b}_{11} / b_{01}$ & $\widehat{b}_{12} / b_{01}$ & $\delta_{1}$ & $\widehat{a}_{11}$ & $\widehat{a}_{12}$ & $\widehat{b}_{11} / b_{01}$ & $\widehat{b}_{12} / b_{01}$ & $\delta_{1}$ \\
\hline \multicolumn{11}{|c|}{$\delta_{n s}=5.24 \%$} \\
\hline 1000 & 1.2829 & -0.3333 & 0.04046 & 0.0312 & 0.0463 & 1.2206 & -0.2576 & 0.03881 & 0.0294 & 0.1204 \\
\hline 1500 & 1.2937 & -0.3465 & 0.04243 & 0.0319 & 0.0341 & 1.2310 & -0.2660 & 0.04077 & 0.0307 & 0.1080 \\
\hline 2000 & 1.3044 & -0.3668 & 0.04270 & 0.0318 & 0.0174 & 1.2508 & -0.2892 & 0.03945 & 0.0292 & 0.0859 \\
\hline 2500 & 1.3108 & -0.37960 .04317 & 0.0321 & 0.0069 & 1.2497 & -0.3004 & 0.03994 & 0.0306 & 0.0795 & \\
\hline 3000 & 1.3051 & -0.3837 & 0.04537 & 0.0329 & 0.0076 & 1.2452 & -0.2948 & 0.04043 & 0.0309 & 0.0848 \\
\hline 3500 & 1.3106 & -0.3886 & 0.04387 & 0.0326 & 0.0026 & 1.2519 & -0.3050 & 0.03951 & 0.0296 & 0.0760 \\
\hline 4000 & 1.3088 & -0.3874 & 0.04428 & 0.0325 & 0.0040 & 1.2653 & -0.3211 & 0.03799 & 0.0290 & 0.0608 \\
\hline True & 1.3139 & -0.3886 & 0.04308 & 0.0315 & 0 & 1.3139 & -0.3886 & 0.04308 & 0.0315 & 0 \\
\hline \multicolumn{11}{|c|}{$\delta_{n s}=16.25 \%$} \\
\hline 1000 & 1.3571 & -0.4624 & 0.04853 & 0.0332 & 0.0625 & 1.2116 & -0.2357 & 0.04010 & 0.0355 & 0.1372 \\
\hline 1500 & 1.3879 & -0.4842 & 0.04813 & 0.0291 & 0.0564 & 1.2484 & -0.3041 & 0.04230 & 0.0356 & 0.0781 \\
\hline 2000 & 1.3657 & -0.4458 & 0.04578 & 0.0284 & 0.0564 & 1.2484 & -0.3041 & 0.04230 & 0.0356 & 0.0781 \\
\hline 2500 & 1.3526 & -0.4311 & 0.04367 & 0.0292 & 0.0419 & 1.2645 & -0.3127 & 0.04389 & 0.0348 & 0.0661 \\
\hline 3000 & 1.3376 & -0.4124 & 0.04375 & 0.0299 & 0.0245 & 1.2572 & -0.2923 & 0.04404 & 0.0336 & 0.0815 \\
\hline 3500 & 1.3205 & -0.3900 & 0.04372 & 0.0307 & 0.0049 & 1.2643 & -0.3020 & 0.04445 & 0.0339 & 0.0728 \\
\hline 4000 & 1.3215 & -0.3931 & 0.04462 & 0.0319 & 0.0066 & 1.2598 & -0.2947 & 0.04617 & 0.0342 & 0.0791 \\
\hline True & 1.3139 & -0.3886 & 0.04308 & 0.0315 & 0 & 1.3139 & -0.3886 & 0.04308 & 0.0315 & 0 \\
\hline \multicolumn{11}{|c|}{$\delta_{n s}=35.68 \%$} \\
\hline 1000 & 1.3992 & -0.5185 & 0.04740 & 0.0281 & 0.1135 & 1.1806 & -0.2297 & 0.04310 & 0.0360 & 0.1503 \\
\hline 1500 & 1.3538 & -0.4715 & 0.04400 & 0.0315 & 0.0671 & 1.2033 & -0.2523 & 0.04599 & 0.0379 & 0.1208 \\
\hline 2000 & 1.3469 & -0.4702 & 0.04385 & 0.0320 & 0.0642 & 1.2176 & -0.2774 & 0.04675 & 0.0379 & 0.1208 \\
\hline 2500 & 1.3384 & -0.4488 & 0.04311 & 0.0330 & 0.0474 & 1.2041 & -0.2608 & 0.04882 & 0.0376 & 0.1230 \\
\hline 3000 & 1.3001 & -0.3976 & 0.04310 & 0.0339 & 0.0121 & 1.2190 & -0.2733 & 0.05037 & 0.0374 & 0.1091 \\
\hline 3500 & 1.3077 & -0.4069 & 0.04492 & 0.0336 & 0.0142 & 1.2457 & -0.3086 & 0.04919 & 0.0358 & 0.0769 \\
\hline 4000 & 1.3081 & -0.3999 & 0.04468 & 0.0336 & 0.0095 & 1.2304 & -0.2865 & 0.04844 & 0.0368 & 0.0963 \\
\hline True & 1.3139 & -0.3886 & 0.04308 & 0.0315 & 0 & 1.3139 & -0.3886 & 0.04308 & 0.0315 & 0 \\
\hline
\end{tabular}

TABLE 2: Identification results of the linear block in the second branch under different $\delta_{n s}$.

\begin{tabular}{|c|c|c|c|c|c|c|c|c|c|c|}
\hline \multirow[b]{2}{*}{$t$} & \multicolumn{5}{|c|}{ The proposed method } & \multicolumn{5}{|c|}{ The method in [18] } \\
\hline & $\widehat{a}_{21}$ & $\widehat{a}_{22}$ & $\widehat{b}_{21} / b_{02}$ & $\widehat{b}_{22} / b_{02}$ & $\delta_{2}$ & $\widehat{a}_{21}$ & $\widehat{a}_{22}$ & $\widehat{b}_{21} / b_{02}$ & $\widehat{b}_{22} / b_{02}$ & $\delta_{2}$ \\
\hline \multicolumn{11}{|c|}{$\delta_{\mathrm{ns}}=5.24 \%$} \\
\hline 1000 & 1.5968 & -0.6984 & 0.0269 & 0.0230 & 0.0872 & 1.6151 & -0.7697 & 0.0399 & 0.0236 & 0.1312 \\
\hline 1500 & 1.4904 & -0.4911 & 0.0322 & 0.0247 & 0.0566 & 1.6166 & -0.7531 & 0.0384 & 0.0242 & 0.1225 \\
\hline 2000 & 1.5532 & -0.6055 & 0.0384 & 0.0235 & 0.0262 & 1.6164 & -0.7662 & 0.0428 & 0.0204 & 0.1374 \\
\hline 2500 & 1.5523 & -0.6090 & 0.0359 & 0.0220 & 0.0271 & 1.6270 & -0.7668 & 0.0453 & 0.0219 & 0.1332 \\
\hline 3000 & 1.5546 & -0.6151 & 0.0364 & 0.0228 & 0.0308 & 1.6070 & -0.7248 & 0.0424 & 0.0227 & 0.1046 \\
\hline 3500 & 1.5092 & -0.5639 & 0.0370 & 0.0248 & 0.0122 & 1.6120 & -0.7092 & 0.0381 & 0.0226 & 0.0980 \\
\hline 4000 & 1.5136 & -0.5715 & 0.0352 & 0.0245 & 0.0070 & 1.5787 & -0.6453 & 0.0359 & 0.0240 & 0.0543 \\
\hline True & 1.5218 & -0.5778 & 0.0305 & 0.0254 & 0 & 1.5218 & -0.5778 & 0.0305 & 0.0254 & 0 \\
\hline \multicolumn{11}{|c|}{$\delta_{\mathrm{ns}}=16.25 \%$} \\
\hline 1000 & 1.4821 & -0.5452 & 0.0313 & 0.0296 & 0.0316 & 1.5969 & -0.7754 & 0.0406 & 0.0271 & 0.1400 \\
\hline 1500 & 1.5003 & -0.5707 & 0.0298 & 0.0274 & 0.0140 & 1.5781 & -0.7581 & 0.0408 & 0.0289 & 0.1162 \\
\hline 2000 & 1.4947 & -0.5452 & 0.0282 & 0.0257 & 0.0261 & 1.5701 & -0.7379 & 0.0397 & 0.0292 & 0.1029 \\
\hline 2500 & 1.5278 & -0.5978 & 0.0291 & 0.0259 & 0.0128 & 1.5695 & -0.7046 & 0.0399 & 0.0296 & 0.0853 \\
\hline 3000 & 1.5312 & -0.6073 & 0.0300 & 0.0265 & 0.0190 & 1.5422 & -0.6779 & 0.0410 & 0.0263 & 0.0631 \\
\hline 3500 & 1.5381 & -0.6077 & 0.0270 & 0.0262 & 0.0210 & 1.5503 & -0.7009 & 0.0421 & 0.0253 & 0.0779 \\
\hline 4000 & 1.5444 & -0.6136 & 0.0259 & 0.0244 & 0.0262 & 1.5477 & -0.7011 & 0.0414 & 0.0261 & 0.0777 \\
\hline True & 1.5218 & -0.5778 & 0.0305 & 0.0254 & 0 & 1.5218 & -0.5778 & 0.0305 & 0.0254 & 0 \\
\hline \multicolumn{11}{|c|}{$\delta_{\mathrm{ns}}=35.68 \%$} \\
\hline 1000 & 1.4290 & -0.4060 & 0.0328 & 0.0267 & 0.1199 & 1.6486 & -0.7773 & 0.0259 & 0.0202 & 0.1520 \\
\hline 1500 & 1.4438 & -0.4203 & 0.0304 & 0.0273 & 0.1080 & 1.6435 & -0.7560 & 0.0261 & 0.0210 & 0.1327 \\
\hline 2000 & 1.4701 & -0.4830 & 0.0306 & 0.0282 & 0.0663 & 1.6275 & -0.7539 & 0.0279 & 0.0222 & 0.1262 \\
\hline 2500 & 1.4441 & -0.4681 & 0.0275 & 0.0272 & 0.0826 & 1.6128 & -0.7248 & 0.0269 & 0.0223 & 0.1062 \\
\hline 3000 & 1.4605 & -0.4875 & 0.0291 & 0.0279 & 0.0671 & 1.6134 & -0.7176 & 0.0229 & 0.0217 & 0.1028 \\
\hline 3500 & 1.4787 & -0.5369 & 0.0324 & 0.0274 & 0.0365 & 1.6137 & -0.7232 & 0.0236 & 0.0219 & 0.1057 \\
\hline 4000 & 1.4846 & -0.5424 & 0.0318 & 0.0273 & 0.0316 & 1.6224 & -0.7396 & 0.0261 & 0.0212 & 0.1171 \\
\hline True & 1.5218 & -0.5778 & 0.0305 & 0.0254 & 0 & 1.5218 & -0.5778 & 0.0305 & 0.0254 & 0 \\
\hline
\end{tabular}



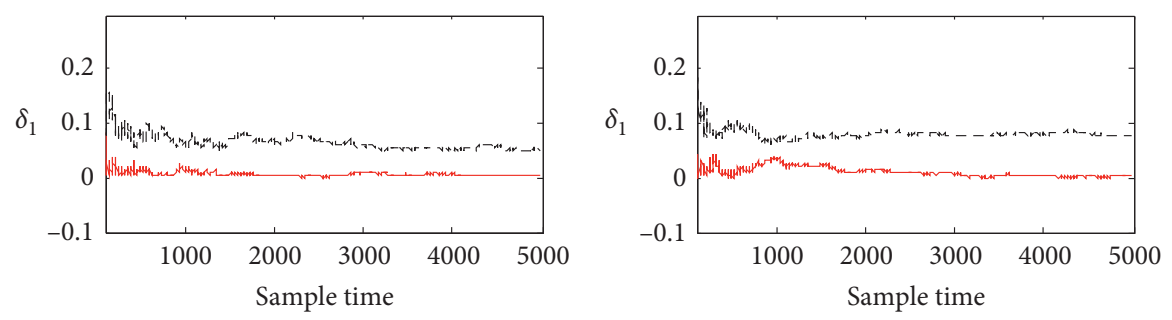

- The proposed method

- - . The method in [18]

(a)

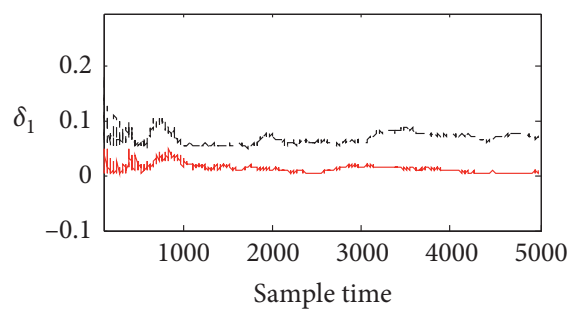

- The proposed method

- - . The method in [18]

(b)

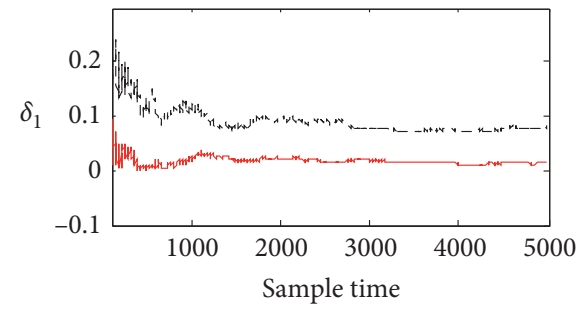

The proposed method

- - . The method in [18]

(c)

c)

The proposed method

-... The method in [18]

(d)

FIGURE 4: The parameter estimation errors of the linear block in the first branch under different $\delta_{\mathrm{ns}}:(\mathrm{a}) \delta_{\mathrm{ns}}=7.33 \%$; (b) $\delta_{\mathrm{ns}}=14.13 \%$; (c) $\delta_{\mathrm{ns}}=22.64 \%$; (d) $\delta_{\mathrm{ns}}=31.47 \%$.

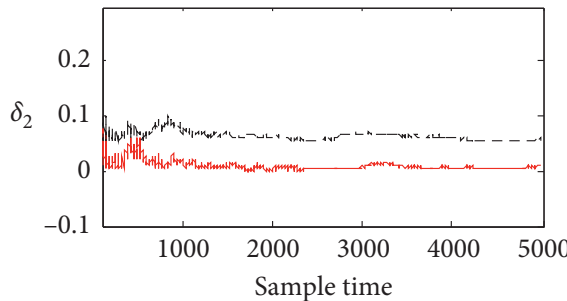

- The proposed method

- - . The method in [18]

(a)

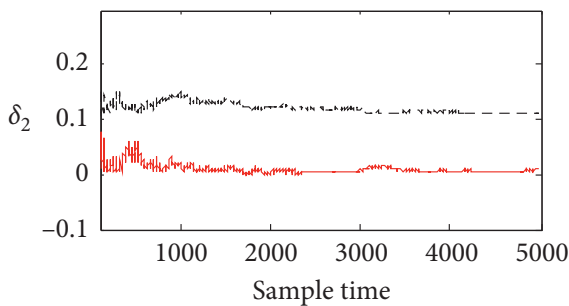

- The proposed method

- - . The method in [18]

(c)

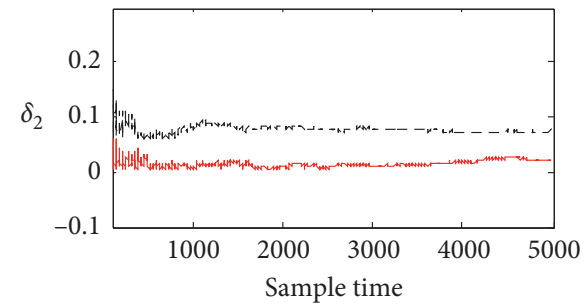

- The proposed method

- - The method in [18]

(b)

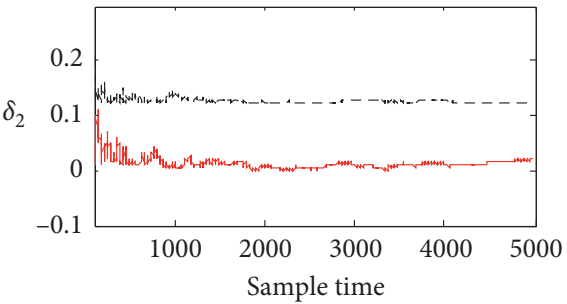

- The proposed method

-.-. The method in [18]

(d)

Figure 5: The parameter estimation errors of the linear block in the second branch under different $\delta_{\mathrm{ns}}$ : (a) $\delta_{\mathrm{ns}}=7.33 \%$; (b) $\delta_{\mathrm{ns}}=14.13 \%$; (c) $\delta_{\mathrm{ns}}=22.64 \%$; (d) $\delta_{\mathrm{ns}}=31.47 \%$.

and $\widehat{v}_{2}(t)=-0.2859 \widehat{v}_{2}(t-1)+0.5980 u_{2}(t-1)$, and the modifying coefficients are $b_{01}=E(y(t) v(t)) / E(v(t) v(t))=$ 0.9536 and $b_{02}=E(y(t) v(t)) / E(v(t) v(t))=0.5680$, respectively. The estimation errors of two linear blocks are $3.66 \times 10^{-2}$ and $2.18 \times 10^{-2}$, respectively.
Next, the design parameters are set as: $S_{01}=0.9991$, $\rho_{1}=1.991, \lambda_{1}=0$ and $S_{02}=0.995, \rho_{1}=1.85, \lambda_{1}=0$, resulting in two neurofuzzy models whose rules are 113 and 55 , respectively. Then, the weights $\mathbf{W}_{1}$ and $\mathbf{W}_{2}$ of two neurofuzzy models are obtained by using (30) and (31). 


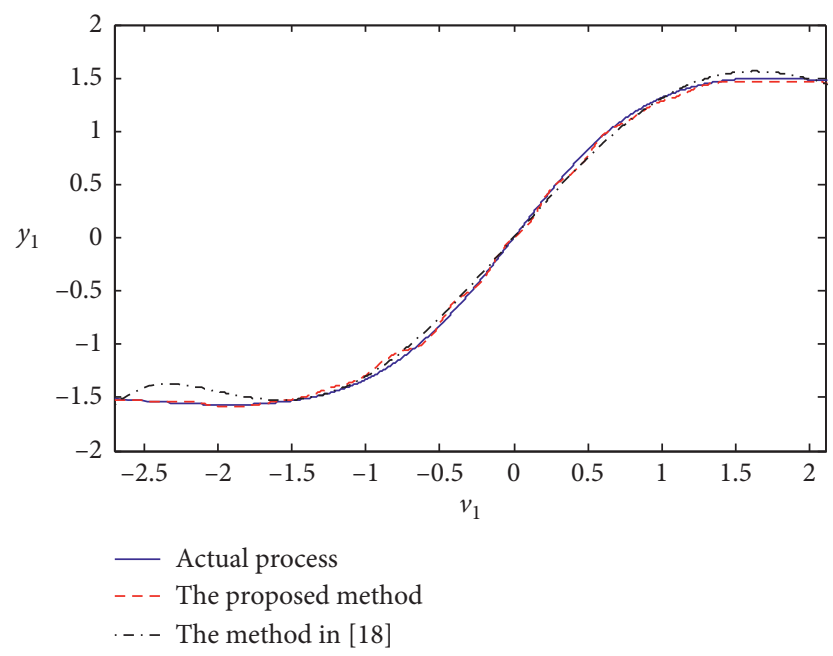

Figure 6: The static nonlinear block in the first branch.

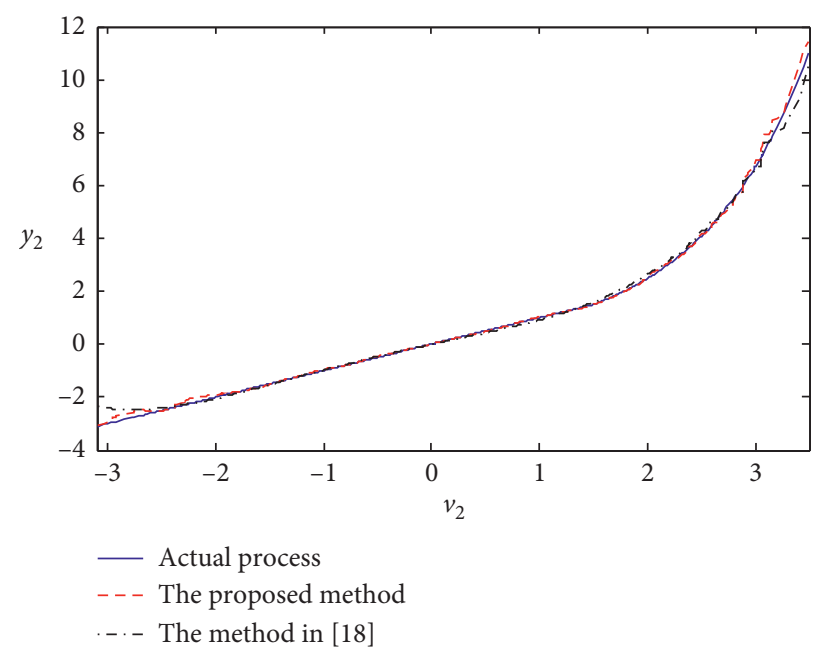

FIgURE 7: The static nonlinear block in the second branch.
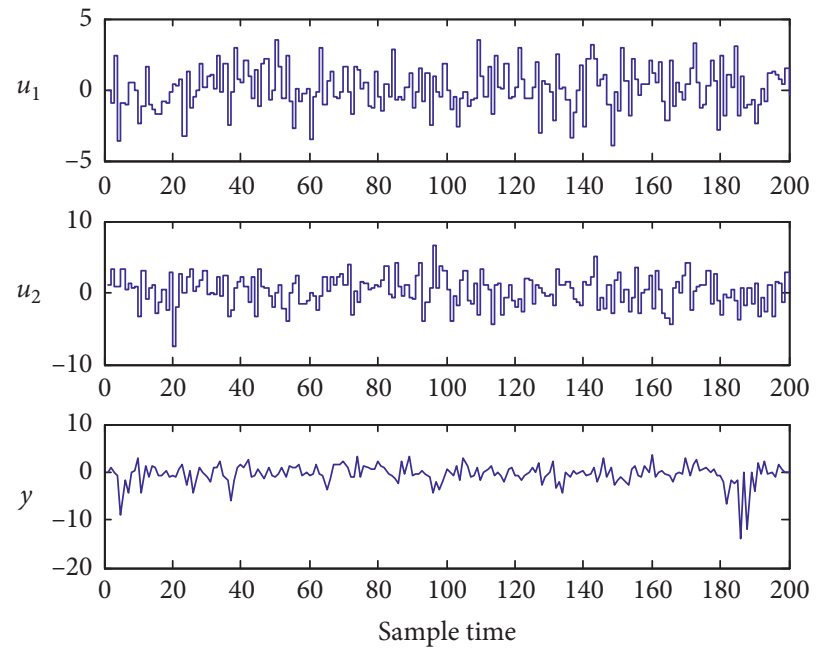

FIgUre 8: Part of the training data used in Example 2. 
TABLE 3: MSEs of various polynomial-based TISO Wiener models.

\begin{tabular}{lccccc}
\hline & $\begin{array}{c}\text { The nonlinear block in the first branch } \\
\text { Order }\end{array}$ & MSE (training) & MSE (validation) & Order & $\begin{array}{r}\text { The nonlinear block in the second branch } \\
\text { MSE (training) }\end{array}$ \\
\hline 7 & $6.01 \times 10^{-2}$ & $7.12 \times 10^{-2}$ & 8 & $6.79 \times 10^{-1}$ & MSE (validation) \\
12 & $4.31 \times 10^{-2}$ & $6.78 \times 10^{-2}$ & 17 & $5.98 \times 10^{-1}$ & $6.56 \times 10^{-1}$ \\
16 & $3.31 \times 10^{-2}$ & $5.34 \times 10^{-2}$ & 24 & $4.03 \times 10^{-1}$ & $6.02 \times 10^{-1}$ \\
19 & $1.24 \times 10^{-2}$ & $3.80 \times 10^{-2}$ & 32 & $2.10 \times 10^{-1}$ & $4.15 \times 10^{-1}$ \\
\hline
\end{tabular}

TABLE 4: MSEs of the proposed TISO Wiener model with different fuzzy rules.

\begin{tabular}{lcccc}
\hline & \multicolumn{2}{c}{ The nonlinear block in the first branch } & \multicolumn{2}{c}{ The nonlinear block in the second branch } \\
The number of fuzzy rules & MSE (training) & MSE (validation) & The number of fuzzy rules & MSE (training) \\
MSE (validation) \\
\hline 45 & $5.80 \times 10^{-3}$ & $8.20 \times 10^{-3}$ & 20 & $2.96 \times 10^{-3}$ \\
90 & $4.03 \times 10^{-3}$ & $3.80 \times 10^{-2}$ & 33 & $3.30 \times 10^{-3}$ \\
113 & $3.79 \times 10^{-3}$ & $4.90 \times 10^{-3}$ & 45 & $1.80 \times 10^{-3}$ \\
122 & $5.40 \times 10^{-3}$ & $6.30 \times 10^{-3}$ & 55 & $8.60 \times 10^{-2}$ \\
\hline
\end{tabular}

TABLE 5: MSEs of the best polynomial-based and the proposed TISO Wiener model.

\begin{tabular}{|c|c|c|c|c|c|}
\hline \multicolumn{3}{|c|}{ The nonlinear block in the first branch } & \multicolumn{3}{|c|}{ The nonlinear block in the second branch } \\
\hline Model & MSE (training) & MSE (validation) & Model & MSE (training) & MSE (validation) \\
\hline Neurofuzzy & $3.79 \times 10^{-3}$ & $4.90 \times 10^{-3}$ & Neurofuzzy & $1.09 \times 10^{-3}$ & $2.70 \times 10^{-3}$ \\
\hline Polynomial & $1.24 \times 10^{-2}$ & $3.80 \times 10^{-2}$ & Polynomial & $2.10 \times 10^{-1}$ & $4.15 \times 10^{-1}$ \\
\hline
\end{tabular}

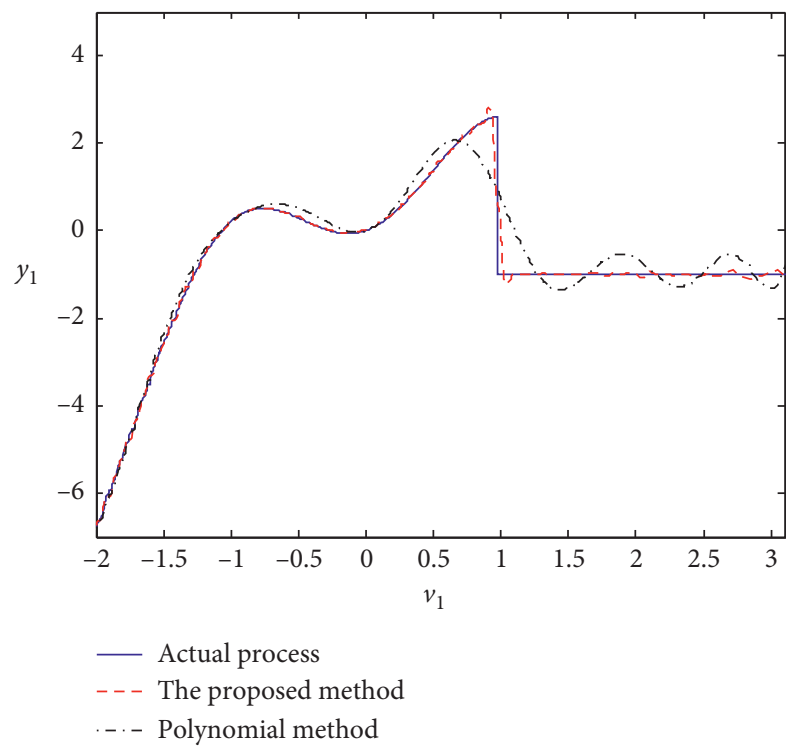

Figure 9: The static nonlinear block in the first branch.

For comparison purpose, the polynomial-based TISO Wiener model is constructed by using the identical training data. Table 3 shows the MSEs for different polynomials based models in two branches; the models with orders of 19 and 32 are chosen to be the best models of two subsystems, respectively. The MSEs of the proposed TISO Wiener model based on different fuzzy rules are summarized in Table 4, and the two neurofuzzy models with fuzzy rules of 113 and 55 are proved to be the

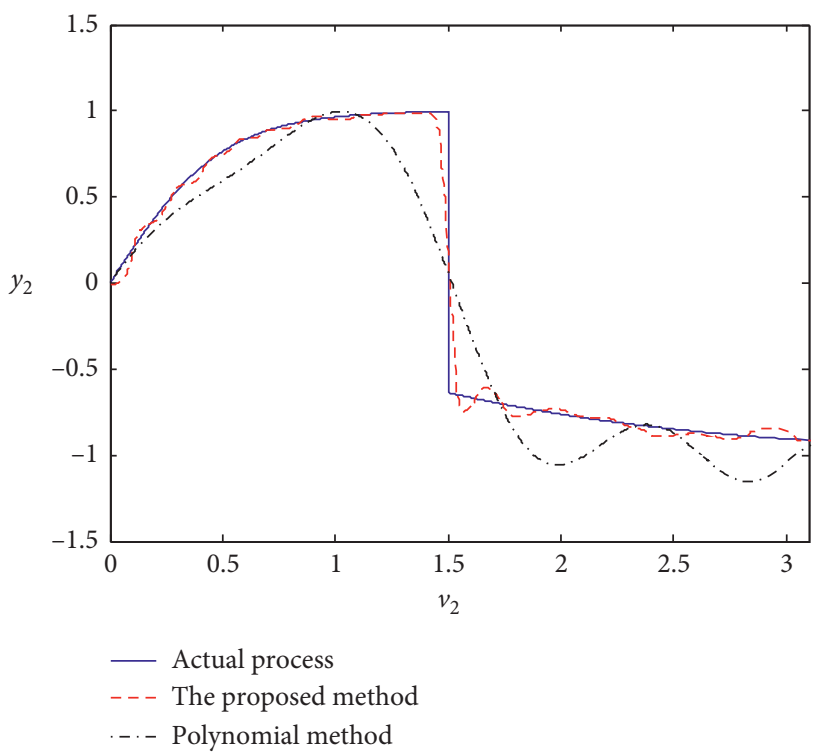

FIGURE 10: The static nonlinear block in the second branch.

best models. Table 5 compares the MSEs for both training and validation data obtained by the best neurofuzzybased model and the best polynomial-based model, respectively. Figures 9 and 10 show the approximation results of the nonlinearities of two branches. As described in Tables 3-5 as well as Figures 9 and 10, the neurofuzzybased TISO Wiener model can achieve better approximation of nonlinearities than the polynomial-based TISO Wiener model. 


\section{Conclusion}

This paper extends Bussgang's classic theorem to a MISO Wiener system and proposes a novel identification method for the correlation analysis algorithm-based MISO Wiener model with noise. At first, several sets of Gaussian signals are adopted to identify the MISO Wiener model, resulting in the identification problem of the dynamic linear blocks separated from that of the static nonlinear blocks. Next, correlation analysis is employed to estimate the parameters of the linear blocks. Finally, least squares algorithm-based identification method of static nonlinear block is presented for the MISO Wiener model. The proposed approach can not only avoid the influence caused by white noise but also solve the problem of initialization and convergence of the model parameters discussed in the existing iterative method used for the identification of the MISO Wiener model. Examples demonstrate the effectiveness of the proposed algorithm.

\section{Data Availability}

The data used to support the findings of this study are included within the article.

\section{Conflicts of Interest}

The authors declare that they have no conflicts of interest.

\section{Acknowledgments}

This work was supported by the National Natural Science Foundation of China (61773251) and Shanghai Science Technology Commission (15510722100, 16111106300, and 17511109400).

\section{References}

[1] A. Kalafatis, N. Arifin, L. Wang, and W. R. Cluett, "A new approach to the identification of $\mathrm{pH}$ processes based on the Wiener model," Chemical Engineering Science, vol. 50, no. 23, pp. 3693-3701, 1995.

[2] A. Harudeen and D. Banjerpongchai, "Nonlinear model predictive control with Wiener model and laguerre function for CSTR process," in Proceedings of the 8th Electrical Engineering/ Electronics, Computer, Telecommunications and Information Technology (ECTI) Association of Thailand (Conference 2011), pp. 557-580, IEEE, Khon Kaen, Thailand, May 2011.

[3] H.-T. Zhang, H.-X. Li, and G. Chen, "Dual-mode predictive control algorithm for constrained Hammerstein systems," International Journal of Control, vol. 81, no. 10, pp. 1609-1625, 2008.

[4] H. H. J. Bloemen, C. T. Chou, T. J. J. V. D. Boom, V. Verdult, M. Verhaegen, and T. C. Backx, "Wiener model identification and predictive control for dual composition control of a distillation column," Journal of Process Control, vol. 11, no. 6, pp. 601-620, 2011.

[5] D. Wang, Z. Zhang, and B. Xue, "Decoupled parameter estimation methods for Hammerstein systems by using filtering technique," IEEE Access, vol. 6, pp. 66612-66620, 2018.
[6] F. Li, J. Li, and D. G. Peng, "Identification method of neurofuzzy-based Hammerstein model with coloured noise," IET Control Theory \& Applications, vol. 11, no. 17, pp. 3026-3037, 2017.

[7] D. Wang, L. Li, Y. Ji, and Y. Yan, "Model recovery for Hammerstein systems using the auxiliary model based orthogonal matching pursuit method," Applied Mathematical Modelling, vol. 54, pp. 537-550, 2018.

[8] D. Wang, Y. Yan, Y. Liu, and J. Ding, "Model recovery for Hammerstein systems using the hierarchical orthogonal matching pursuit method," Journal of Computational and Applied Mathematics, vol. 345, pp. 135-145, 2019.

[9] D. Wang and F. Ding, "Parameter estimation algorithms for multivariable Hammerstein CARMA systems," Information Sciences, vol. 355-356, pp. 237-248, 2016.

[10] P. Wachel and G. Mzyk, "Direct identification of the linear block in Wiener system," International Journal of Adaptive Control and Signal Processing, vol. 30, no. 1, pp. 93-105, 2015.

[11] R. Raich, G. T. Zhou, and M. Viberg, "Subspace based approaches for Wiener system identification," IEEE Transactions on Automatic Control, vol. 50, no. 10, pp. 1629-1634, 2005.

[12] J. C. Gómez and E. Baeyens, "A combined SVM/OB-based wiener model identification method," IFAC Proceedings Volumes, vol. 45, no. 16, pp. 638-643, 2012.

[13] D. Q. Wang, H. B. Liu, and F. Ding, "Highly efficient identification methods for dual-rate Hammerstein systems," IEEE Transactions on Control Systems Technology, vol. 23, no. 5, pp. 1952-1960, 2015.

[14] S. V. Vaerenbergh, J. Vía, and I. Santamaría, “Adaptive kernel canonical correlation analysis algorithms for nonparametric identification of Wiener and Hammerstein systems," EURASIP Journal on Advances in Signal Processing, vol. 2008, no. 1, Article ID 875351, 2008.

[15] D. Wang and F. Ding, "Least squares based and gradient based iterative identification for Wiener nonlinear systems," Signal Processing, vol. 91, no. 5, pp. 1182-1189, 2011.

[16] L. Vanbeylen and R. Pintelon, "Blind maximum-likelihood identification of wiener and Hammerstein nonlinear block structures," in Lecture Notes in Control and Information Sciences, vol. 404, pp. 273-291, Springer, Berlin, Germany, 2010.

[17] Y. Chen, Z. Y. Liu, and H. J. Zhang, "Research and simulation of Volterra series kernel identification on Wiener model," in Proceedings of the International Conference on Environmental Science and Information Application Technology, vol. 4, pp. 95-98, IEEE, Wuhan, China, July 2010.

[18] G. Fibich and G. Papanicolaou, "A new identification method for Wiener and Hammerstein systems," Physics Letters A, vol. 239, no. 3, pp. 167-173, 2004.

[19] K. Tiels, P. S. C. Heuberger, and J. Schoukens, "Parameter reduction of MISO Wiener-Schetzen models using the best linear approximation," vol. 8443, no. 8, pp. 2114-2211, 2012.

[20] D. Feroldi, J. C. Gomez, and V. Roda, "Identification of PEM fuel cells based on support vector regression and orthonormal bases," in Proceedings of the IEEE International Symposium on Intelligent Control, pp. 1-6, IEEE, Kolkata, India, October 2016.

[21] L. Zhou, X. Li, and F. Pan, "Gradient-based iterative identification for MISO Wiener nonlinear systems: application to a glutamate fermentation process," Applied Mathematics Letters, vol. 26, no. 8, pp. 886-892, 2013.

[22] M. M. D. Silva, "Prediction error identification of minimally parameterized Wiener models in anesthesia," IFAC Proceedings Volumes, vol. 44, no. 1, pp. 5615-5620, 2011. 
[23] A. Naitali, F. Giri, E. Elayan, and M. Haloua, "Biosocial culture inspired hierarchical algorithm for MISO block oriented nonlinear system identification: application to ozone modelling," IFAC Proceedings Volumes, vol. 41, no. 2, pp. 7433-7438, 2008.

[24] J. J. Bussgang, "Crosscorrelation functions of amplitudedistorted Gaussian signals," Technical Report 216, MIT Research Laboratory of Electronics, Cambridge, MA, USA, 1952.

[25] A. H. Nuttall, Theory and Application of the Separable Class of Random Processes, Massachusetts Institute of Technology, Cambridge, MA, USA, 1958.

[26] M. Enqvist and L. Ljung, "Linear approximations of nonlinear FIR systems for separable input processes," Automatica, vol. 41, no. 3, pp. 459-473, 2005.

[27] W. Greblicki and M. Pawlak, "Nonparametric identification of Hammerstein systems," IEEE Transactions on Information Theory, vol. 35, no. 2, pp. 409-418, 1989.

[28] I. Goethals, L. Hoegaerts, V. Verdult, J. A. Suykens, and B. D. Moor, "Subspace identification of Hammerstein-Wiener systems using kernel canonical correlation analysis," 2004.

[29] L. Jia, X. Li, and M.-S. Chiu, "Correlation analysis based MIMO neuro-fuzzy Hammerstein model with noises," Journal of Process Control, vol. 41, pp. 76-91, 2016.

[30] L. Jia, X. Li, and M.-S. Chiu, "The identification of neuro-fuzzy based MIMO Hammerstein model with separable input signals," Neurocomputing, vol. 174, pp. 530-541, 2016.

[31] F. Li, L. Jia, D. Peng, and C. Han, "Neuro-fuzzy based identification method for Hammerstein output error model with colored noise," Neurocomputing, vol. 244, pp. 90-101, 2017.

[32] L. Jia, Q. Xiong, and F. Li, "Correlation analysis method based SISO neuro-fuzzy Wiener model," Journal of Process Control, vol. 58, pp. 73-89, 2017.

[33] C. L. Cham, A. H. Tan, and W. H. Tan, "Identification of a multivariable nonlinear and time-varying mist reactor system," Control Engineering Practice, vol. 63, pp. 13-23, 2017.

[34] M. Jafari, M. Salimifard, and M. Dehghani, "Gradient based iterative identification of multivariable Hammerstein-Wiener models with application to a Steam Generator Boiler," in Proceedings of the 20th Iranian Conference on Electrical Engineering (ICEE 2012), pp. 916-921, IEEE, Tehran, Iran, May 2012.

[35] A. Haryanto and K. S. Hong, “"Maximum likelihood identification of Wiener-Hammerstein models," Mechanical Systems \& Signal Processing, vol. 41, no. 1-2, pp. 54-70, 2013.

[36] M. Ławryńczuk, "Identification of Wiener models for dynamic and steady-state performance with application to solid oxide fuel cell," Asian Journal of Control, vol. 21, no. 4, pp. 1836-1846, 2019.

[37] I. Benyó, J. Kovács, J. Mononen, and U. Kortela, "Modelling of steam temperature dynamics of a superheater," International Journal of Simulation, vol. 6, no. 6, pp. 3-9, 2005.

[38] A. Haryanto, A. Turnip, and K. S. Hong, "Parameter identification of a superheater boiler system based on WienerHammerstein model using maximum likelihood method," in Proceedings of the 2009 7th Asian Control Conference, pp. 1346-1351, IEEE, Hong Kong, China, August 2009.

[39] S. M. Ross, Introduction to Probability Models, Academic Press, Cambridge, MA, USA, 10th edition, 2011.

[40] L. Ljung, System Identification: Theory for the User, Tsinghua University Press, Beijing, China, 2002.

[41] L. Jia, M.-S. Chiu, and S. S. Ge, "A noniterative neuro-fuzzy based identification method for Hammerstein processes," Journal of Process Control, vol. 15, no. 7, pp. 749-761, 2005.
[42] L. Jia, J. P. Shi, and M. S. Chiu, "Fuzzy neural model with global convergence for batch process," Information \& Control, vol. 38, no. 6, pp. 685-691, 2009. 


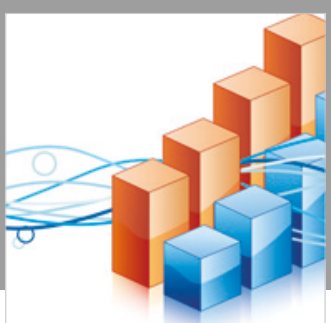

Advances in

Operations Research

\section{-n-m}
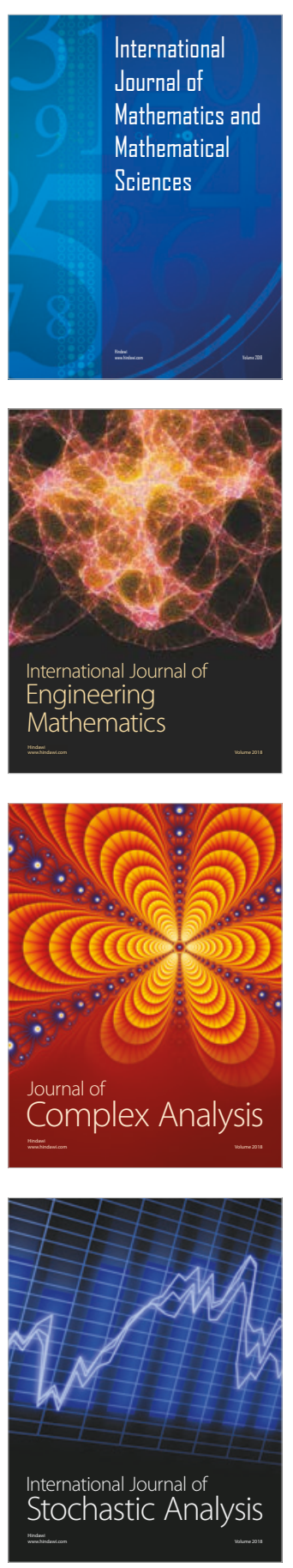
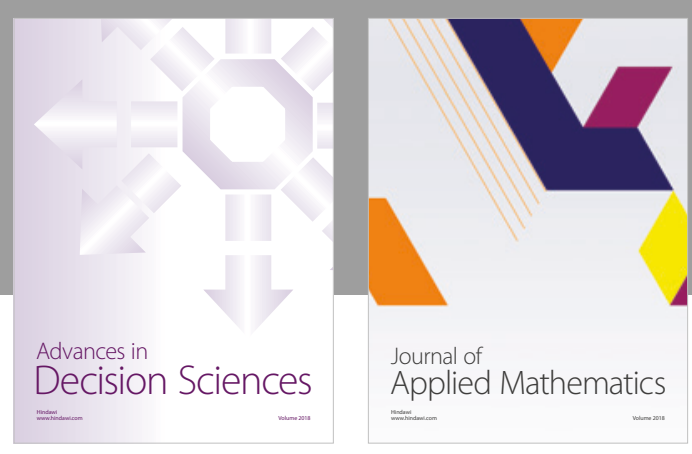

Journal of

Applied Mathematics
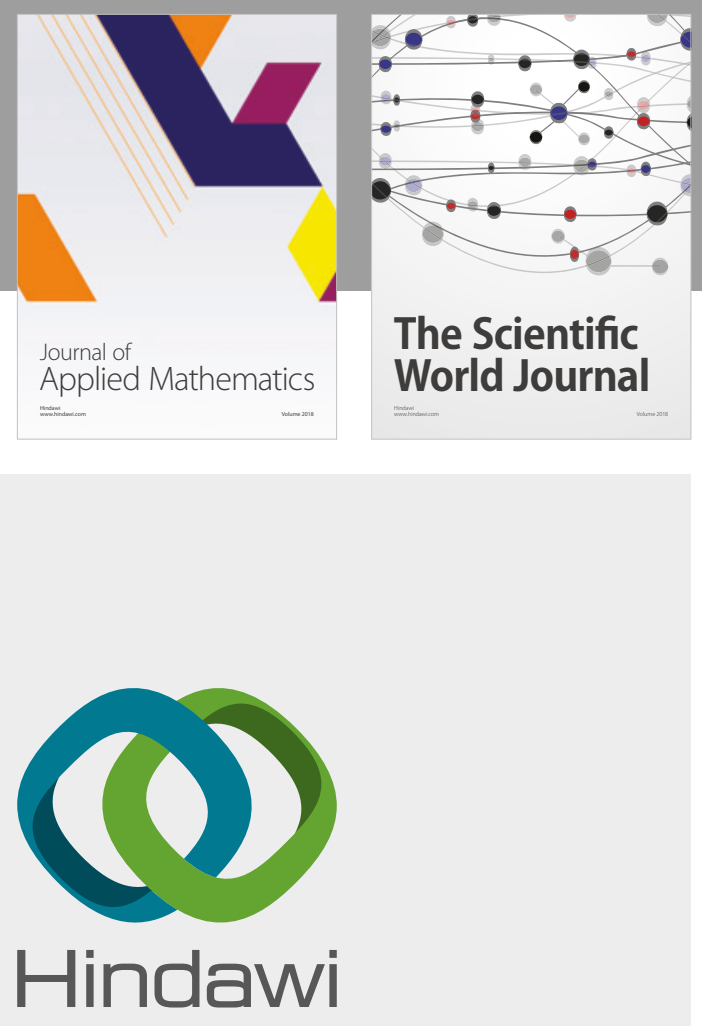

Submit your manuscripts at

www.hindawi.com

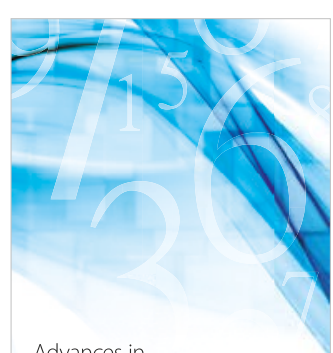

Advances in
Numerical Analysis
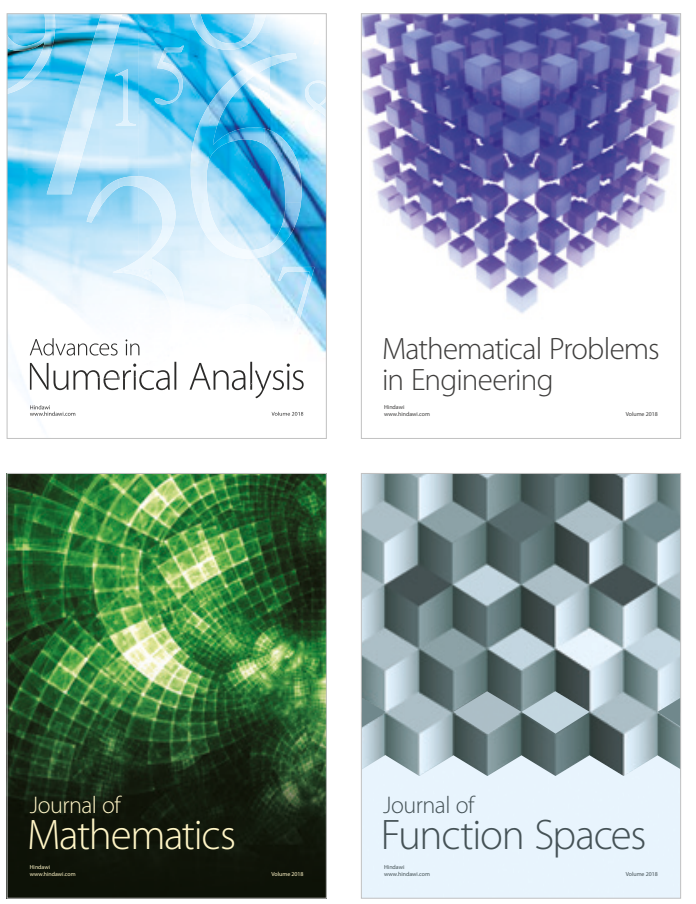

Mathematical Problems in Engineering

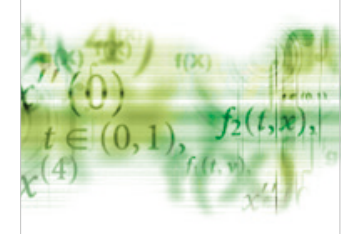

International Journal of

Differential Equations

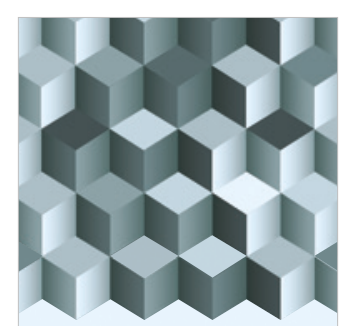

Journal of

Function Spaces
The Scientific

World Journal

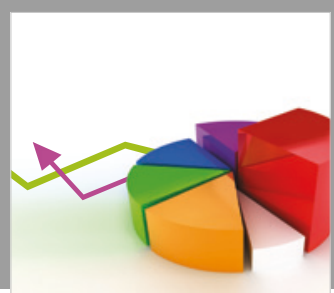

Journal of

Probability and Statistics
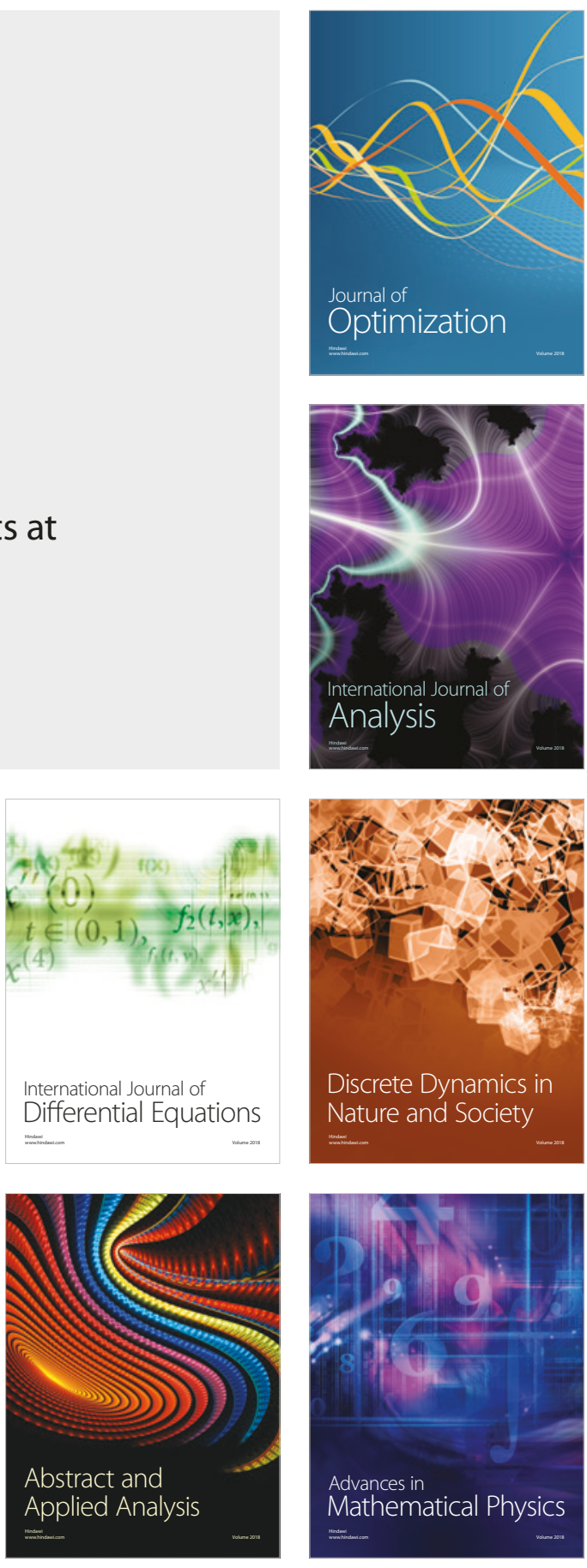